\title{
A Flexible Robotic Arm with Hydraulic Skeleton*
}

\author{
Hitoshi KIMURA**, Mokutaro KATAOKA**, Shotaro SUZUKI***, \\ Daisuke AKIMOTO ${ }^{\dagger}$ and Norio INOU ${ }^{* *}$ \\ ** Tokyo Institute of Technology, Department of Mechanical and Control Engineering \\ 2-12-1, Ookayama, Meguro-ku, Tokyo 152-8552, Japan \\ E-mail: kimura@mech.titech.ac.jp \\ *** $\mathrm{IHI}$ Corporation \\ TOYOSU IHI BUILDING., 1-1, Toyosu 3-chome, Koto-ku, Tokyo 135-8710, Japan \\ $\dagger$ Toshiba Corporation \\ 1-1, Shibaura 1-chome, Minato-ku, Tokyo 105-8001, Japan
}

\begin{abstract}
This study aims to build a totally flexible mechanical system with hydraulic skeleton driving mechanism. The main components of this system are two types of flexible bags. One is a structural bag with constant inner pressure. The other is an actuator bag with controlled inner pressure. Such flexible system will provide various advantages, for example, safety, portability, lightweight and dealing with fragile objects. Efficiency is also an advantage because only actuator parts consume driving fluid with the proposed system. This paper deals with a flexible robotic arm as an example of the proposed mechanism. With geometric relationship, effective parts arrangement and driving force at the joint are discussed in this paper. Quantitative estimations of structural deformation and driving force of flexible bags are important for design of the proposed system. However, numerical analysis of flexible bags is difficult because of their large nonlinear deformation. This study tries to analyze large nonlinear deformations of flexible bags with the nonlinear finite element analysis software ABAQUS. From the analytical results, this paper discusses unique effects of structural deformation depending on the driving force. The validity of the analysis is verified by experimental results. Both results show that the effect of concave deformation of a structural bag at the joint is 10 times larger than normal cantilever deformation. Pick and place motion of a raw egg is also performed with a proposed robotic arm avoiding large concave deformation without complex control such as force sensing and so on.
\end{abstract}

Key words : Soft Mechanism, Fluid Power Systems, Finite Element Method, Nonlinear Problem, Pneumatic Equipment

\section{Introduction}

Flexible mechanism with soft materials has several advantages owing to passive deformation. For example, the flexible parts greatly decrease physical damage of collision accidents. A soft gripper can readily grasp a fragile object in a controlled fashion. Terrain adaptive shape change is also a performance advantage of flexible mechanisms against the environments such as a narrow space ${ }^{(1)}$.

This study proposes a hydraulic skeleton driving mechanism to realize innovative flexibility. The hydraulic skeletons are found in living things such as worm and actinia. A simple example of a hydraulic skeleton is shown in Fig.1. This element can transform its shape and stiffness by changing the inner pressure. Lightweight is an advantage of the proposed mechanism when the air is used as the driving fluid. In addition, a depressurized hydraulic skeleton can be compactly folded. It also means advantages in storage and portability. Of course, flexible mechanisms are not suit to fast, powerful and dynamic motion compared with

${ }^{*}$ Received 5 June, 2012 (No. 12-0250) [DOI: 10.1299/jamdsm.6.1107]

Copyright (c) 2012 by JSME 
rigid mechanisms, these advantages are deserving of esteem. Almost all conventional flexible mechanisms include hard parts at joints, bases, and so on ${ }^{(2),(3)}$. However, the proposed mechanism is able to configure structural parts and actuator parts flexible without any hard and heavy parts.

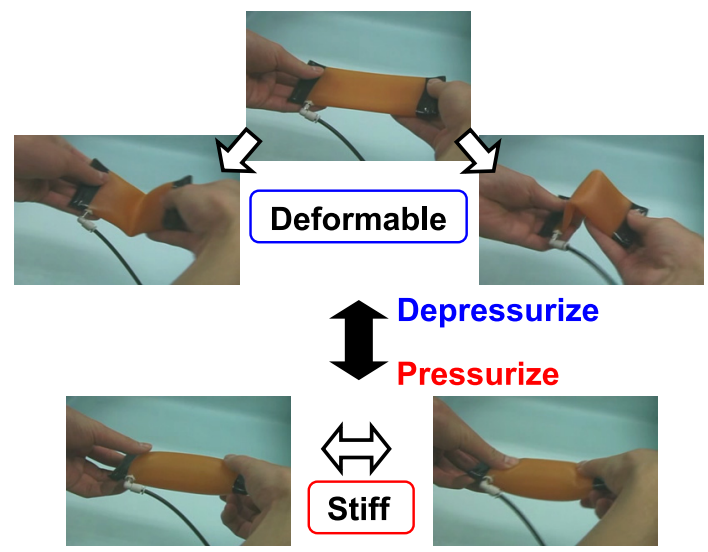

Fig. 1 Deformation of a hydraulic skeleton with pressure change

The advantages of such extreme flexibility are noteworthy, however, there are few practical applications because of difficulties caused by flexibility. The main problem is estimation of large deformation of flexible parts. In general, estimation of such large deformation is not easy because of nonlinearity. Moreover, bending angle of proposed actuator is more than $90^{\circ}$. Such deformation is extremely large compared with Mckibben Artificial Muscles ${ }^{(4),(5)}$.

This paper deals with a new flexible robotic arm as a particular example application. The robotic arm is composed of two types of air bags. One works as a structural part with constant inner pressure. The other is an actuator with controlled inner pressure to generate driving force as the joint torque. This functional division improves the driving efficiency because only actuator parts consume driving fluid compared with conventional flexible mechanisms ${ }^{(6)-(8)}$.

This study aims to establish a design method of extremely flexible mechanism. Although there are lots of nonlinear analyses of lumped structure ${ }^{(9),(10)}$, there are only a few studies of large deformations of hollow and compliant structures with limited conditions ${ }^{(11),(12)}$. However, in these days, with development of computer technology, it has become possible to calculate large deformation with nonlinear Finite Element Analysis (FEA). If the analyses have a certain validity, it will be a strong design tool of flexible mechanism. Although authors already tried to analyze some large deformation problems of flexible bags, the discussion for practical application is not enough because the analyses only deal with the stress concentration and the actuator torque ${ }^{(13)}$. Detailed geometry of the parts at the joint is described for the purpose of actual driving force estimation. Operating range of the joint is also derived by the geometric relation. In addition, this study also discusses the relationship between structural deformation and driving force with the nonlinear FEA software ABAQUS. Because of the flexibility, structural deformation is not negligible compared with conventional rigid link mechanisms. From the analytical results, this paper describes unique effects of structural concave deformation. The validity of the analyses are verified by experiments with the flexible bags and prototype flexible arm.

\section{Operating principle of a hydraulic skeleton driving mechanism}

\subsection{Structure of a flexible robotic arm}

A schematic diagram of the proposed flexible arm is illustrated in Fig.2. The robotic system can be configured totally flexible as shown in the figure. The arm is mainly composed of serial-linked structural bags and actuator bags arranged at the joints. When a flexible bag is pressurized, it becomes stiffer like an inflated balloon forming a specific shape. As mentioned in the previous section, the inner pressure of the structural bags is kept constant to maintain the 
form of the links. In contrast, the inner pressure of the actuator bags is controlled to generate and to regulate the driving forces.

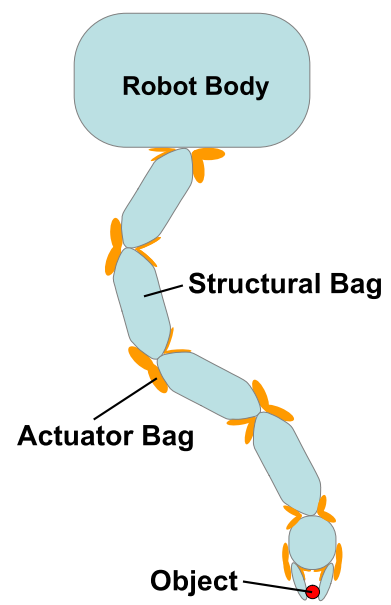

Fig. 2 A basic concept of proposed robotic arm

When the air is used as the driving fluid, the total weight of robotic arm which is made of urethane rubber sheets becomes extremely light. A long serial linked arm will benefit from the lightweight. Moreover, compared to other conventional long serial linked arms such as a wire driven $\operatorname{arm}^{(14)}$, the proposed system will easily realize S-shape motion with simple structure (Fig.3).

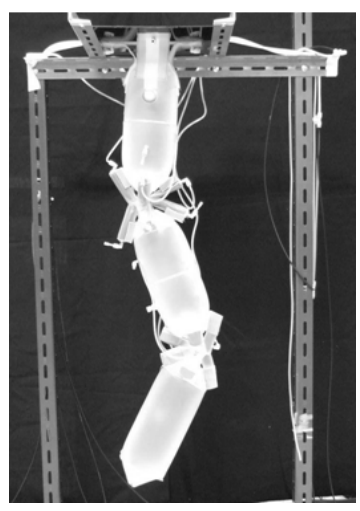

Fig. 3 S-shape motion of 3-link arm

On the other hand, if the driving fluid is water, the specific gravity of the arm can easily be set near 1. In general, to adjust the specific gravity of the mechanism is not easy for under water applications. In addition, using water contributes not only a provision of the driving fluid but also the pressure resistant structure.

The proposed flexible system is also easy to realize a 2-DOF active joint link mechanism with more than 3 actuator bags at the joint as shown in Fig.4. When the all bags are depressurized, the mechanism can be folded into very compact. 


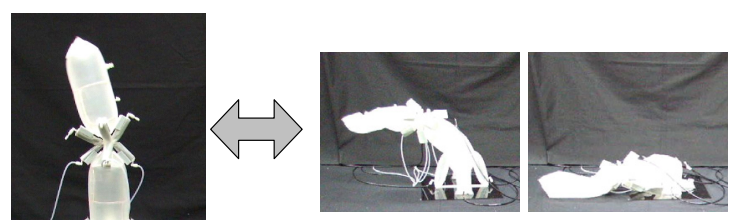

14. Depressurize all parts

$\rightarrow$ advantage of portability \& storage
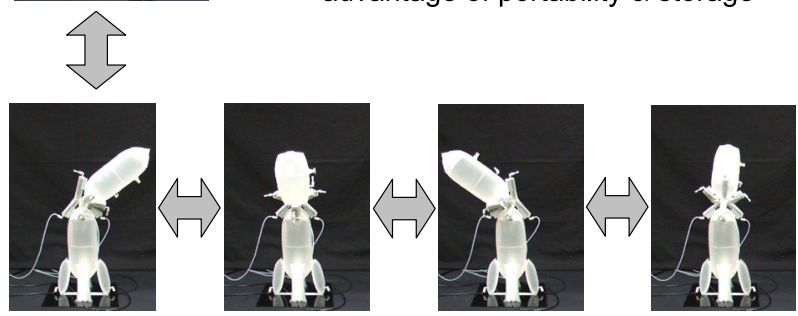

2DOF joint with simple mechanism

Fig. 4 Prototype 2-link robotic arm with 2-DOF active joint

\subsection{Geometry of a joint}

There are several unique issues to build the proposed robotic arm because of flexibility and inflated structure. The arrangement of parts is one of the issues. Geometry of the structural bags and the actuator bags directly affect the performance of the arm. Geometric discussion is necessary to design such flexible mechanisms. For simplicity, the following discussion deals with 1-DOF active joint. The discussion of 1-DOF joint can be applied to the foregoing 2DOF joint. Fig.5 illustrates a geometrical relationship between two structural bags and one actuator bag at the joint. From Fig.5, the joint operating angle (joint movable angle) $\alpha$ is represented in the following equation,

$$
\alpha=\pi-\left(\varphi_{\mathrm{s}}+2 \varphi_{\mathrm{a}}\right)
$$

where $\varphi_{s}$ is the taper angle at the end of the structural bag, $\varphi_{a}$ is the corresponding angle of the actuator bag which occupy a part of the operating angle $\alpha$. From the equation (1), when $\varphi_{s}$ or $\varphi_{a}$ is large, initial bending angle $\theta_{a}$ of the actuator bag becomes large. This means that large torque configuration causes small operating angle $\alpha$ because the torque of the actuator bag has positive correlation with the bending angle $\theta_{a}{ }^{(13)}$. In contrast, if $\varphi_{s}$ or $\varphi_{a}$ is small, the initial bending angle $\theta_{a}$ becomes small and the initial torque decreases compared with the case mentioned above. This is a trade-off problem. The distance between the joint and the actuator $l_{j a}$ is also an important parameter. In practical mechanism, however, it is difficult to set $l_{j a}$ as 0 due to inflated structures (Fig.5). This causes nonlinearity of the driving force. If the length of $l_{j a}$ becomes large, the actuator bag cannot transmit its driving force to the structural bags. Proper parts fixation is important for this mechanism.

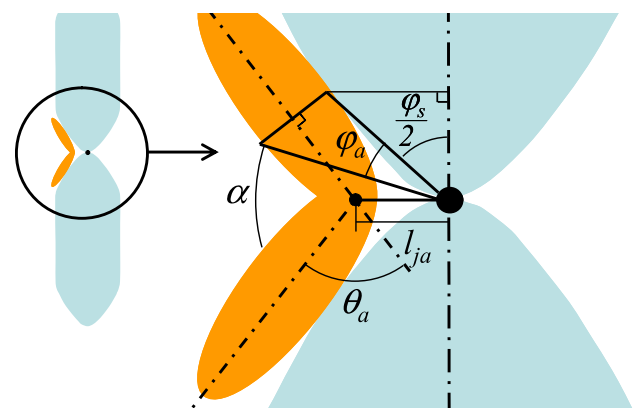

Fig. 5 Geometric relationship at a joint of proposed arm 


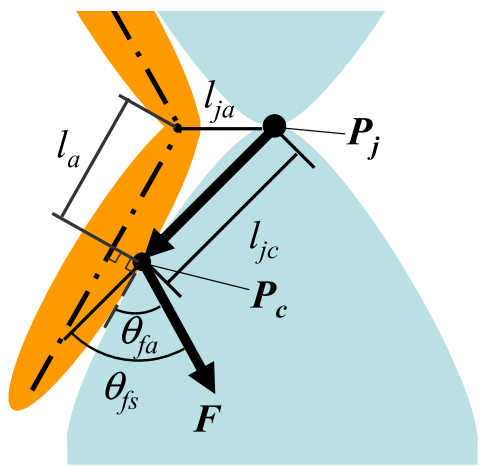

Fig. 6 Parts arrangement and driving force

The placement of the actuator bag will substantially affect the driving force and the efficiency. The geometry of driving force is drawn in Fig.6. In actual, the contact part between the actuator bag and the structural bag is not a point but an area. Following discussion is an approximation. The direction of the driving force vector $\boldsymbol{F}$ is depending on the several parameters e.g. $l_{j a}, l_{j c}$ and so on. From the cross product of position vector $\left(\boldsymbol{P}_{\boldsymbol{c}}-\boldsymbol{P}_{j}\right)$ and the force vector $\boldsymbol{F}$, the reduction ratio $r$ is described by the following equation,

$$
r=\frac{T_{k}}{T_{a}}=\frac{l_{j c} \sin \theta_{f s}}{l_{a} \sin \theta_{f a}}(\geq 1)
$$

where $T_{k}$ is the torque at the joint, $T_{a}$ is the torque at the rotation axis of actuator bag, $l_{j c}$ is the distance between the joint axis and the contact point of the structural bag and the actuator bag, $l_{a}$ is the corresponding length of the actuator bag, $\theta_{f a}$ is the angle between the center line of the actuator bag and the force vector $\boldsymbol{F}$, and $\theta_{f s}$ is the angle between $l_{j c}$ and $\boldsymbol{F}$.

From the equation (2), the joint torque $T_{k}$ depends nonlinearly on the actuator bag torque $T_{a}$. When the distance of two axes $l_{j a}$ is equal to 0 , two approximations $\theta_{f a} \approx \theta_{f s}$ and $l_{a} \approx l_{j c}$ can be realized. These conditions mean the relation of $T_{k} \simeq T_{a}$ (see eq.(2)). To keep the transmission efficiency, the value of $l_{j a}$ should be kept small. From the geometric relation, the difference between $\theta_{f a}$ and $\theta_{f s}$ becomes the maximum when the bending angle of the actuator bag $\theta_{a}=0$. When $\theta_{a}$ is almost equal to 0 , the difference $\theta_{f a}-\theta_{f s}$ becomes an important factor to estimate the driving force. In actual fact, $l_{j c} \sin \theta_{f s}$ is always larger than $l_{a} \sin \theta_{f a}$. Therefore, the reduction ratio $r>1$ with the proposed joint. Because of flexible structure, $l_{a}$, $\theta_{f a}, \theta_{f s}$ and $l_{j} c$ are not constant and the ratio $r$ is variable.

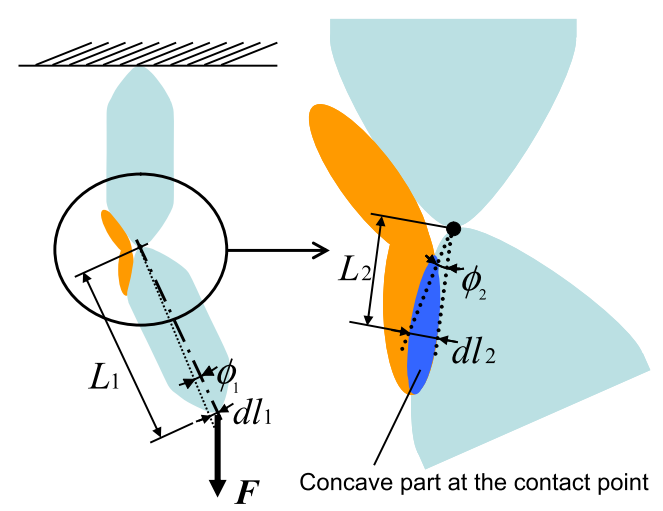

Fig. 7 Deformations of a structural bag related to motion of the arm

With respect to the structural bag, there are two types of deformations $\left(d l_{1}, d l_{2}\right)$ in the arm as drawn in Fig.7. One is a cantilever deformation $d l_{1}$ of structural bag caused by a load $\boldsymbol{F}$ at the end point of the arm. The other is a concave deformation $d l_{2}$ caused by the actuator bag at the contact point of the actuator bag and the structural bag (Fig.7). In conventional 
studies of manipulator systems with flexible links ${ }^{(15),(16)}$, such concave deformation is not discussed because the joint parts are defined as a rigid body. With proposed flexible arm, however, $d l_{2}$ directfy affects the link angle. In addition, such deformation affects the contact state between actuator and structural bags. If there is a certain load which causes $d l_{2}$ deformation, the bending angle $\theta_{a}$ and torque $T_{a}$ of the actuator bag increase because of the load direction. Besides, $l_{a}$ and $l_{j c}$ (in Fig.6) also decrease simultaneously. From these geometrical relationship, the reduction ratio $r$ also increases in this situation. As a result, joint torque $T_{k}$ increases and the joint supports large load which causes $d l_{2}$ deformation. In this situation, the deformation of actuator bag is smaller than that of structural bag because the inner pressure of the actuator bag is higher than the structural bag. In general, it is quite difficult to have a quantitative discussion about a contact problem between two flexible objects. The effects of two types of deformations $\left(d l_{1}, d l_{2}\right)$ are discussed in the next session with analytical and experimental results.

\section{Mechanical analysis of the flexible mechanism}

\subsection{Nonlinear finite element analysis of structural bags}

Required analyses are the deformation of the structural bag depending on the driving force of the actuator bag. Based on the previous discussion, this study tries to estimate quantitative behavior of the proposed arm with nonlinear FEA software ABAQUS. Compared with conventional stiff mechanism, the structural deformation can not be neglected with the proposed flexible mechanism. In former studies, there are some reports of large deformation dealing with buckling of a pipe structure ${ }^{(17),(18)}$, however, there is almost none of similar numerical studies of extremely large deformation of flexible bags.

This study analyzes models consist of shell elements which have a material property of urethane rubber with $0.3 \mathrm{~mm}$ thickness and the inner pressure is $0.005 \mathrm{MPa}$. The inner pressure of the fluid bag is limited depending on the durability. Authors are now trying to make more durable flexible bag, however, this bag can deal with about $0.2 \mathrm{~kg}$ object as mentioned later. At the present stage, this paper deals with the pressure area $0.005 \sim 0.04 \mathrm{MPa}$. Of course, the atmospherical pressure is $0.1 \mathrm{MPa}$. Two type material properties are used for the analysis. One is obtained by experimentally measured result of uniaxial tensile test. The other is biaxial tensile property which is assumed by 1.4 times value of the uniaxial tensile test ${ }^{(19),(20)}$. Material modulus of third-order Ogden model ${ }^{(21),(22)}$ is determined by using both of the material properties. This third-order Ogden model is widely used in nonlinear FEA of rubber materials.

Deformations of a structural bag $d l_{1}$ is analyzed under the usual cantilever configuration as shown in Fig.8. One side of the structural bag is completely fixed. The other side of the structural bag is set free and a loading force is applied on the surface. The analytical model has $200 \mathrm{~mm}$ length and $50 \mathrm{~mm}$ radius.

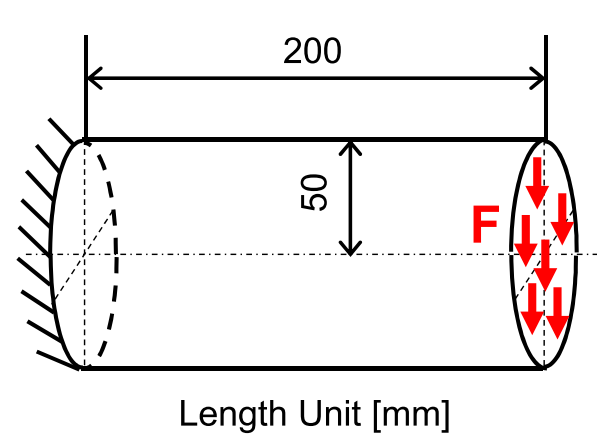

(a) The boundary condition and applied load

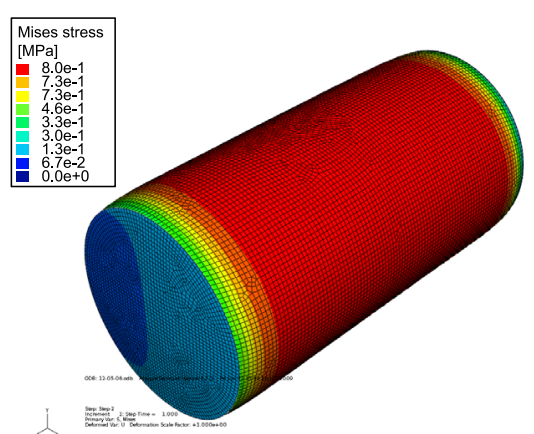

(b) Example of analytical result (stress and deformation)

Fig. 8 FEA of $d l_{1}$ (cantilever bending) 
Next, the concave deformation $d l_{2}$ is also estimated with FEA. Fig.9 denotes the boundary condition and sample result of the analysis. The load $F$ is uniformly set on the generatrix of the cone. Boundary conditions are given so as to have no interference on the bag deformation. The torque is the product of the applied load and the generatrix length as well as the cantilever analysis.

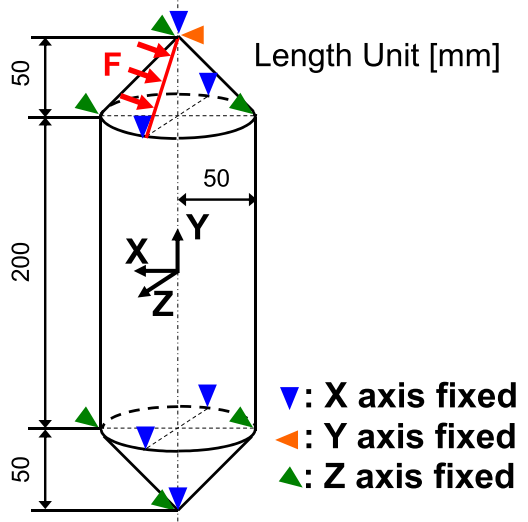

(a) The boundary condition and applied load (b) Example of analytical result (stress and deformation)

Fig. 9 FEA of $d l_{2}$ (partial concave)

To confirm the validity of these analyses, this study also measures actual $d l_{1}$ and $d l_{2}$. A pressure regulator and an indicator are used to control the inner pressure of the fluid bags in this measurement. Fig.10 shows these instruments for the measurement. The configuration of the deformation measurements are denoted in Fig.11.

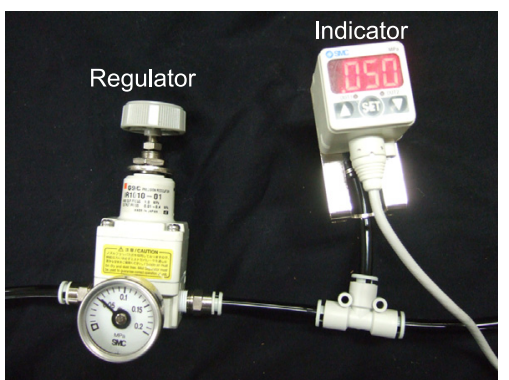

Fig. 10 Pressure regulator and indicator for the actuator control

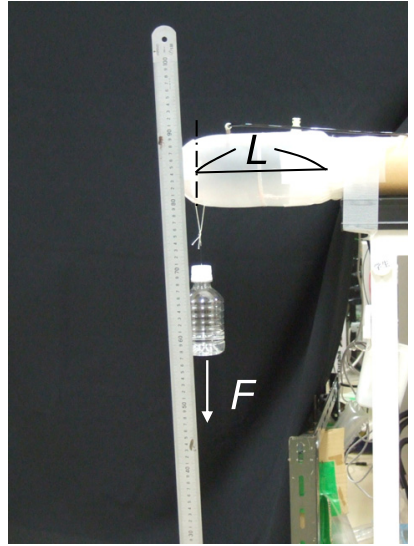

(a) Cantilever deformation $\left(d l_{1}\right)$

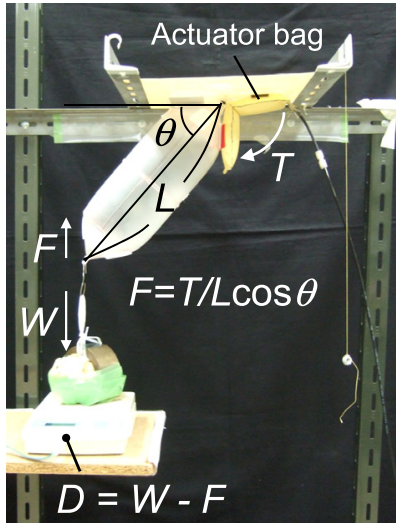

(b) Concave deformation $\left(d l_{2}\right)$

Fig. 11 Deformation measurement of a structural bag 
The measurements are performed so that the load conditions are correspond with the analyses as much as possible. The measurement of $d l_{1}$ is simple (Fig.11(a)). The load is set on the end of the structural bag so as to the link length $L=200 \mathrm{~mm}$. The input pressure of the bag is kept constant at $0.005 \mathrm{MPa}$ as well as the analysis.

In contrast, the measurement of $d l_{2}$ is not easy. With normal balanced situation, the link angle is variable depending on the input torque, however, a constant link angle $\theta$ is necessary to measure the effect of $d l_{2}$. To solve this problem, this study uses a weigher to keep the link angle constant as shown in Fig.11 (b). With this configuration, the forces are always balanced because the torque change is compensated by the weigher. Only the force $D(=W-F)$ changes even if the input torque $T$ is variable. Where $D$ is the force at the weigher, $W$ is a certain constant load and $F(=T / L \cos \theta)$ is the link force. The deformations of the structural bag are measured from high resolution pictures $(2848 \times 2136$ pixels $)$. Joint angle changes caused by both deformations are also measured from the picture by drawing a center line of structural bag on the photograph. The torque of the actuator bag is estimated from the bending angle ${ }^{(13)}$. The method of torque estimation of the actuator bag is described in the next subsection. From these analytical and experimental results, this study estimates the effects of $d l_{1}$ (cantilever deformation) and $d l_{2}$ (concave deformation).

Fig.12 plots the relationship between the input torque and link angle change. From the analytical result, the joint angle change is calculated as follows: 1) The case of $d l_{1}$ : the angle is calculated using the lines through the center of both ends. The angle between two lines, with load and without load, is calculated as the link angle change. 2) The case of $d l_{2}$; the angle is calculated from the generatrix without load and the most concave position with load. Obviously, the angle change depends on the load, the size of bags, and so on. These analyses are performed for the parts of the prototype robotic arm.

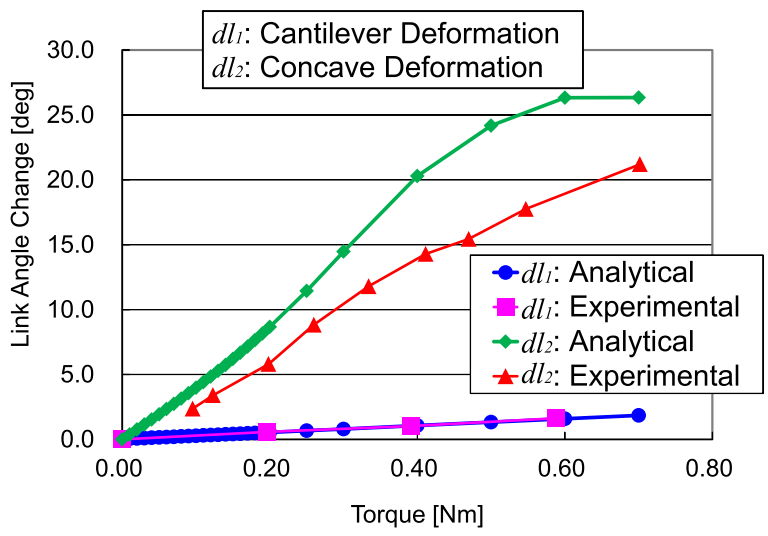

Fig. 12 Link angle change with $d l_{1}$ and $d l_{2}$ depending on the torque

In this graph, analytical results almost agree with the experimental results. Since the analytical configuration of $d l_{2}$ uses a uniform line load (concentrated load), the experimental values of $d l_{2}$ (surface load) are little smaller than analytical results, however, the two results show the same tendency. These results support a certain validity of the analyses. Moreover, the structural deformation of the flexible bags can be estimated with the proposed method. This method can be used for the kinematics, dynamics, path planning and so on.

As shown in the graph, the concave deformation $d l_{2}$ significantly affects the posture of the arm in large torque range. The angle change of $d l_{2}$ is about more than 10 times larger than that of $d l_{1}$. This means that the effect of $d l_{2}$ is not negligible especially when the input torque is large. On the other hand, there is almost no angle change caused by $d l_{1}$ under the same torque. Compared with both deformations, $d l_{2}$ affects the joint angle and the displacement of the link end is amplified by the link length whereas $d l_{1}$ only affects the link end position without any amplification. In general, the deformations of $d l_{1}$ and $d l_{2}$ are of the same order of 
magnitude because they are phenomena on the same bag. Taking into account of these facts, the effect of $d l_{2}$ is always larger than that of $d l_{1}$. However, the problem of $d l_{2}$ is not too serious under a feedback control system because the effect of $d l_{2}$ monotonically increases. In addition, when the input torque is small, $d l_{2}$ is predictable because it is almost linear. Consequently, positional compensation of $d l_{2}$ deformation or avoiding heavy load are necessary for practical application.

\section{Prototype robotic arm}

\subsection{Arm design and the posture management}

To estimate the performance of the robotic arm, mechanical properties of the parts should be known in advance. The deformation of the structural bag is one of important factors for the kinematics of the flexible arm. This study builds a prototype robotic arm with the structural bags which is analyzed in the previous section (Fig.12). For intuitive demonstration, this study tries to realize pick and place motion of a raw egg which is not easy for conventional robotic arm. Including the weight of the end effector, the arm should be capable of lifting at least 0.2 kg. Fig.13 (a) draws a frame format of the prototype arm. As drawn in Fig.13 (b), the effect of $d l_{2}$ is small if the position of the end effector is close to the vertical line from the root of the arm (the origin).

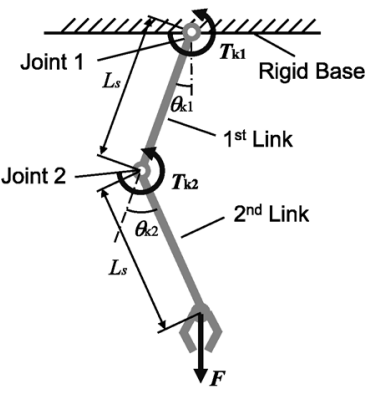

(a) The frame format of the arm

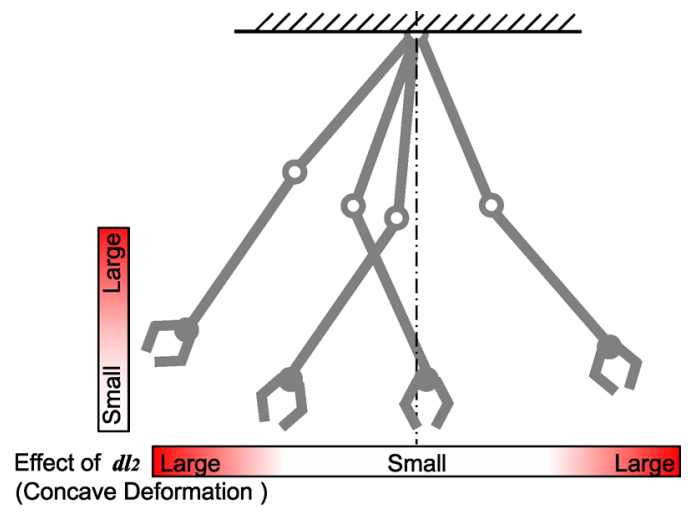

(b) The effect of concave deformation $d l_{2}$

Fig. 13 Arm posture and control difficulty

The effect of $d l_{2}$ described here is based on assumption that it simply depends on the joint torque $T_{k}$. In terms of the arm posture change caused by $d l_{2}$, the effect of $\theta_{k 1}$ is larger than that of $\theta_{k 2}$ because the angle change of the root joint largely affects the arm posture in general. If the arm posture is given, each $d l_{2}$ can be calculated from the $T_{k}$ obtained from the kinematics. If the following parameters are given, $T_{k 1}$ and $T_{k 2}$ are derived by kinematics. The required parameters are the length of structural bag $L_{s}(=300 \mathrm{~mm})$, the load $F$ and the joint angles $\theta_{k 1}, \theta_{k 2}$. On the other hand, the torque of the actuator bag $T_{a}$ can be estimated from the bending angle $\theta_{a}$ by the following empirical equation ${ }^{(13)}$.

$$
T_{a}\left(p, w, \theta_{a}\right)=p w^{3} f\left(\theta_{a}\right) .
$$

Where $f\left(\theta_{a}\right)$ is expressed by the following fifth-order equation.

$$
\begin{aligned}
f\left(\theta_{a}\right) & =1.02 \times 10^{-13} \times \theta_{a}{ }^{5}-3.87 \times 10^{-11} \times \theta_{a}{ }^{4} \\
& +5.51 \times 10^{-9} \times \theta_{a}{ }^{3}-3.61 \times 10^{-7} \times \theta_{a}{ }^{2}+1.01 \times 10^{-5} \times \theta_{a}
\end{aligned}
$$

Each coefficient in the equation (4) is obtained by the analytical results. This equation is only rough approximation because the torque should have hysteresis and so on due to the potential energy. In order to confirm the difference between the joint torque $T_{k}$ and actuator torque $T_{a}$, this study manufactured a prototype robotic arm using the flexible bags. As represented by 
the torque function in the equation (4), the torque is not linear to the bending angle, however, the torque and the input pressure are linearly related. Therefore, the actuator is able to control by the input pressure at the present stage. The torque is also depending on the bag width, appropriate bag shape will improve the controllability. This is one of future works.

With this prototype, the base frame of the arm is made of metal for experimental measurement. The material of structural and actuator bags is $0.3 \mathrm{~mm}$ thickness urethane rubber sheet. The sheets are fabricated by thermal compression bonds with ultrasonic welder. Actuator bag length is $160 \mathrm{~mm}$ and $24 \mathrm{~mm}$ diameter (corresponding to $38 \mathrm{~mm}$ width bag). A structural bag shape is the same of the analytical model (Fig.9). The length of the cylinder part is $200 \mathrm{~mm}$, and the height of the cones is $50 \mathrm{~mm}$. The weight of an actuator bag and a structural bag are $0.01 \mathrm{~kg}$ and $0.045 \mathrm{~kg}$ respectively. Bellows and nylon string are used for the joint to connect two structural bags. Hook and loop fasteners are used for adhesion between structural bag and actuator bag because the fastener has advantages of adhesion, attaching and removing. The joint mechanism at the base plate is also the same. With this configuration, the distance $l_{j a}$ has a certain lower limit. Two actuator bags are arranged at each joint for 1-DOF.

To confirm the arm motion, this study performs a load lifting experiment as shown in Fig.14. The load is changed every $0.05 \mathrm{~kg}$ from 0 to $0.3 \mathrm{~kg}$ taking into account of the predicted load $0.2 \mathrm{~kg}$. The upper joint is joint 1 , and the lower joint is joint 2 . For each $n(n=1,2)$, the joint angle $\theta_{k n}$, and the bending angle of the actuator bag $\theta_{a n}$ are measured by high resolution photographs as well as the previous deformation measurement.

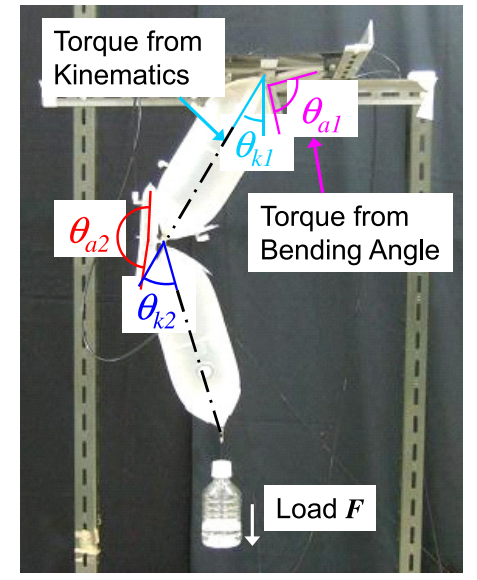

Fig. 14 Arm posture with $0.1 \mathrm{~kg}$ load

Fig.15 represents the measured angles and calculated torques of the two methods. For simplicity, the effect of $d l_{2}$ is not included here. The joint torques are calculated by two methods. The joint torque $T_{k n}$ is calculated from the $\theta_{k n}$ using kinematics. The actuator torque $T_{a n}$ is calculated from the empirical equation (3) and (4) using the bending angle $\theta_{a}$ of the actuator bag. Both tendencies of torque change almost agree with each other. This result supports a certain validity of the empirical equation. In Fig.15(b), $T_{k n}$ is almost always larger than $T_{a n}$ at each joint. It seems to be a contradiction because the input torque $T_{a n}$ should be larger than the required torque $T_{k n}$. However, this result can be explained by the variable reduction ratio $r$ represented in the equation (2). As described before, $T_{k}$ is larger than $T_{a}$ because $r=T_{k} / T_{a}>1$, although it is not easy to measure exact $r$. Therefore, this result does not conflict the principle. Needless to say, the most large reason of the difference of $T_{a}$ and $T_{k}$ is measurement error. Especially, the bending angle of the actuator and the estimation might cause accumulated error. There might be other possible reasons of measurement error. The one is the effect of $d l_{2}$, however, because of the torque estimation and measurement accuracy, it is not clear how does $d l_{2}$ actually affect in this measurement at the present stage. From the experimental result (Fig.12), the joint angle might be affected about $5^{\circ}$ under $0.2 \mathrm{Nm}$. The second is the accuracy of the empirical equation whereas the $T_{k n}$ should have a certain 
accuracy. The third is the hysteresis of the actuator because it is a flexible inflated structure. The result of actuator torque measurement suggests a large influence of hysteresis, however, authors do not have enough data for quantitative discussion yet. Verification of these points is one of important future works.

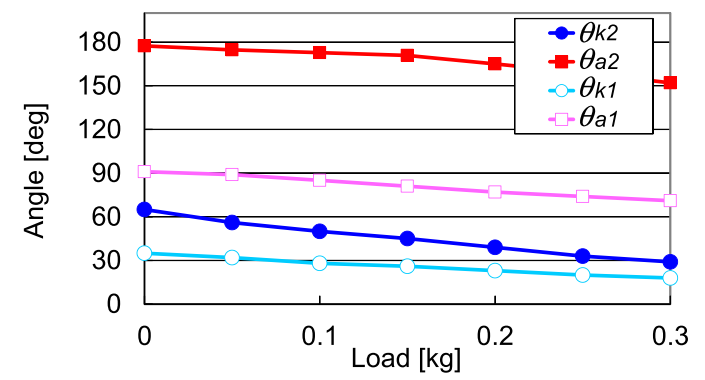

(a) Measured angles of joints and actuator bags from photo

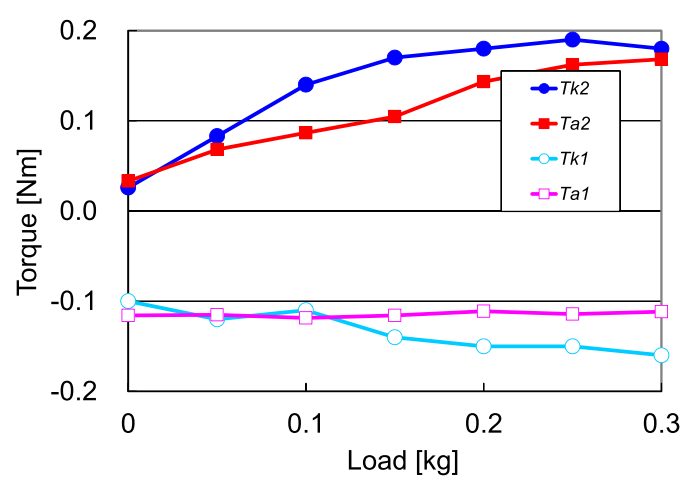

(b) Calculated torque of $T_{k n}$ and $T_{a n}(n=1,2)$

Fig. 15 Torque estimation from arm kinematics and empirical equation

From above discussions, estimations of the structural deformation and the torque from the empirical equation can be used for the design of the flexible mechanism. In terms of dynamic characteristics, the proposed mechanism is not suitable for quick motion which requires dynamic characteristics of the actuator. Vibration and its dumping are very difficult problem with flexible mechanisms in general. Conventional rigid mechanisms will be used for such purpose for the time being.

\subsection{Pick and place motion}

To realize the pick and place motion of a raw egg, this study also manufactured a soft box type gripper. Fig.16 shows the overview of the gripper. The gripper is made of the same material of other bags and the weight is $0.12 \mathrm{~kg}$. It uses rubber strings for release motion at the end of the finger like tips around the mouth. The structure is very flexible and it is easy to grasp a fragile object with this gripper.

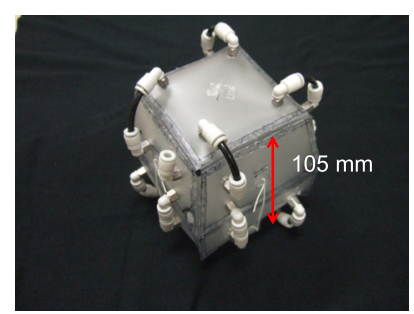

(a) Top view

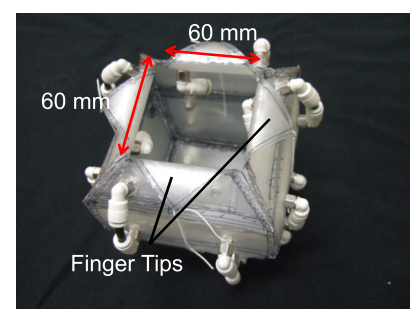

(b) Bottom view

Fig. 16 Soft box type gripper for a fragile object 
Fig.17 demonstrates pick and place motion of a raw egg with the 2-link robotic arm. Each joint has 2-DOF with 4 actuator bags with this arm. Torque of the arm is controlled by regulators shown in Fig.10. Valves are controlled by a microcomputer automatically with this system. As the first step of realizing the motion, the path is planned to keep the required torque less than $0.05 \mathrm{Nm}$ to avoid the effect of $d l_{2}$. With this torque range, the maximum angle change caused by $d l_{2}$ is about less than $2^{\circ}$ (see Fig.12). The position difference of the 1 st link end is about $8 \mathrm{~mm}$ whereas the gripper mouth size is $60 \times 60 \mathrm{~mm}$. The $d l_{2}$ deformation of the 2 nd link is almost negligible because the torque at the 2 nd joint is kept very small with this path planning. However, if large torque is required during the motion, $d l_{2}$ will affect the arm posture. A compensation for $d l_{2}$ such as gain control will be necessary in some way. To develop the strong point of the proposed arm, complex control system is not relevant. Usage requirements should be set with a certain margin of mechanical performance of the flexible arm. Only pressure and valve control, the arm can grasp a raw egg (about $40 \mathrm{~mm}$ diameter $\times 60 \mathrm{~mm}$ length) without complex control such as force sensing, owing to enough flexibility of the gripper. This result supports that the proposed flexible mechanism is suit to deal with fragile objects.
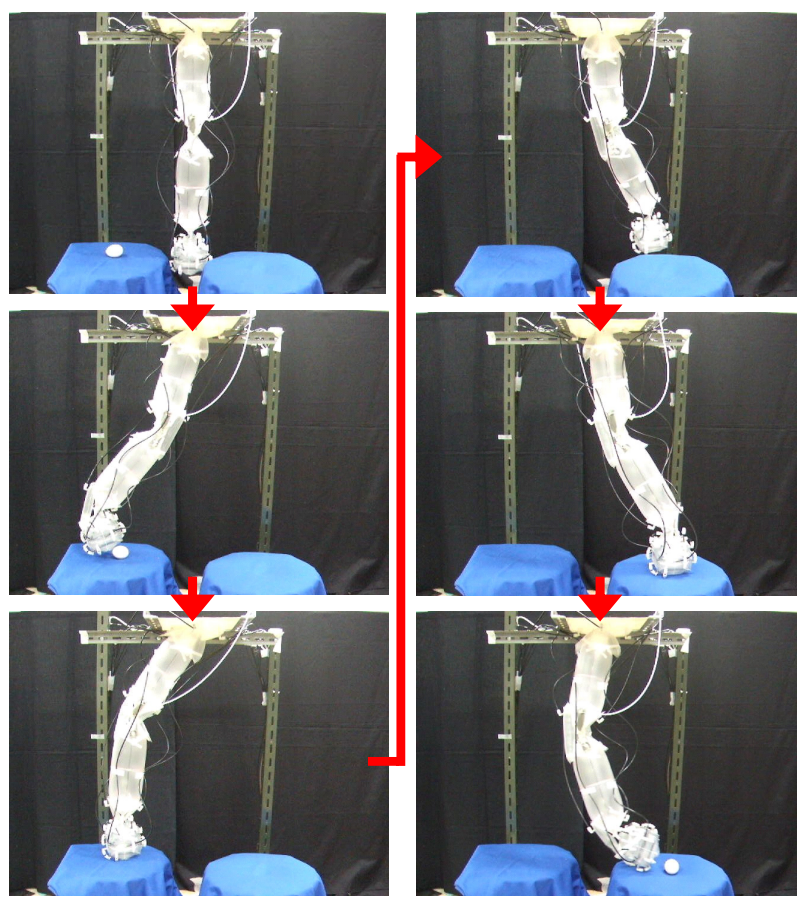

Fig. 17 Pick and place motion of a raw egg with 2-link arm

\section{Conclusion}

This study proposed a new effective flexible robotic arm using hydraulic skeleton driving mechanism with flexible bags. The operating angle and torque reduction ratio are calculated from the geometry at the arm joint. This study also estimates the structural deformation with nonlinear FEA software ABAQUS. Such estimation is difficult in general because the problem includes large nonlinear deformation. Normal cantilever deformation and partial concave deformation are analyzed with this study. Especially, the concave deformation shows a large effect for the link angle. The effect is about 10 times larger than that of the cantilever deformation. The validity of the analyses are verified by an experiment. Empirical equation of the torque of the actuator bag is confirmed by prototype flexible 2-link arm. The estimated torque shows almost agree with the torque of kinematics. More accurate torque measurement of the actuator is one of important future works. With a prototype 2-link robotic arm, pick and place motion of a raw egg is also performed with very simple control. 


\section{References}

( 1 ) H. Kimura, F. Kajimura, D. Maruyama, M. Koseki and N. Inou, Flexible hermeticallysealed mobile robot for narrow spaces using hydraulic skeleton driving mechanism, Proc. IEEE/RSJ International Conf. on Intelligent Robot and Systems, p.4006-4011, (2006)

( 2 ) D. Mishima, T. Aoki, and S. Hirose, Development of a pneumatically controlled expandable arm for rescue searches in tight spaces, International Journal of Robotics Research, Vol.25, No.1, p.103-110, (2006)

( 3 ) Y. Hayakawa and S. R. Pandian, Development of a hybrid element by using sponge core soft rubber actuator, Proc. IEEE International Conf. on Robotics and Automation, p.538-543, (2005)

( 4 ) H. F. Schulte, The characteristics of the McKibben artificial muscle, The Application of External Power in Prosthetics and Orthotics, No.874. p.94-115,(1961).

( 5 ) B. Tondu, P. Lopez, Modeling and control of McKibben artificial muscle robot actuators, IEEE Control System. Vol.20, No.2, p.15-38, (2000).

( 6 ) K. Suzumori and T. Abe, Applying a flexible microactuators to pipeline inspection robots, Proc. IMACS/SICE International Conf. on Robotics and Manufacturing System, p.515-520, (1993).

( 7 ) K. Suzumori T. Maeda, H. Watanabe and T. Hisada, Fiberless flexible microactuator designed by finite-element method, Proc. IEEE/ASME Trans. on Mechatronics, 2, p.281286, (1997)

( 8 ) H. Tsukagoshi, S. Kase, T. Kato, T. Suzuki, A. Kitagawa, Wearable Fluid Power Composed of Flat Tubes to Activate th Body Source, Proc. of the 7th JFPS International Symposium on Fluid Power, 1, p.203-206, (2008)

( 9 ) R. Gracie, J. Oswald, T. Belytschko, On a new extended finite element method for dislocations: Core enrichment and nonlinear formulation, Journal of the Mechanics and Physics of Solids, Vol.56, p.200-214, (2008)

(10) Franck J. Vernerey, Brian Moran, Nonlinear, Large Deformation Finite-Element Beam/Column Formulation for the Study of the Human Spine: Investigation of the Role of Muscle on Spine Stability, Journal of Engineering Mechanics, Vol. 136, No. 11,p.1319-1328, (2010)

(11) D. C. Pamplona, P. B. Goncalves and S. R. X. Lopes, Finite deformations of cylindrical membrane under internal pressure, International Journal of Mechanical sciences, 48, p.683-696, (2006)

(12) Kozulin, A. A., Skripnyak, V. A., Strength cal-culation of polymer pipeline elements, The 8th Russian-Korean International Symposium on Science and Technology KORUS, p.29-32, (2004).

(13) D. Maruyama, H. Kimura, M. Koseki,N. Inou, Driving force and structural strength evaluation of a flexible mechanical system with a hydrostatic skeleton, Journal of Zhejiang University-SCIENCE A, Applied Physics\&Engineering, pp.255-262, (2010).

(14) S. Hirose and R. Chu, Development of a Lightweight Torque Limiting M-Drive Actuator for Hyper-Redundant Manipulator Float Arm, Proc. IEEE International Conf. on Robotics and Automation, p.2831-2836, (1999)

(15) T. Matsuda and S. Murata, Stiffness distribution control-locomotion of closed link robot with mechanical softness, Proc. IEEE International Conf. on Robotics and Automation, p.1491-1498, (2006)

(16) T. Yoshikawa, A. Ohta and K. Kanaoka, State estimation and parameter identification of flexible manipulators based on visual sensor and virtual joint model, Proc. IEEE International Conf. on Robotics and Automation, 3, p.2840-2845, (2001)

(17) T. Itoh and Y. Tomita, Mechanics of structure, Kokuminkagakusya, (1987) (in Japanese)

(18) T. Kawai and Y. Fujitani, Analysis of buckling, Baifukan, (1974) (in Japanese)

(19) Y. Yamashita, Estimation of biaxial tensile curve from uniaxial tensile tests in rubber 
model, Proc. MSC. Software solution forum, 16, (2000) (in Japanese).

(20) T. Nagata, T. Kobayashi and Y. Yamashita, Estimation of biaxial tensile model of rubber material from uniaxial tensile tests, Proc. Japan Society of Mechanical Enfineers Conf. on Computational dynamics, p.55-56, (2001) (in Japanese).

(21) R. W. Ogden, Large deformation isotropic elasticity on the correlation of theory and experiment for incompressible rubberlike solids, Proc. The Royal Society of London, series A, Mathmatical and Physical Sciences, Vol.326 No.1567, p.565-584 (1972)

(22) R. W. Ogden, Nonlinear elastic deformations Dover publications, (1997) 